\title{
Simulation Study and Comparative Evaluation of Viral Contiguous Sequence Identification Tools
}

Cody Glickman ( $\nabla$ cody.glickman@cuanschutz.edu )

University of Colorado Anschutz Medical Campus

Jo Hendrix

University of Colorado Anschutz Medical Campus

Michael Strong

National Jewish Health

\section{Research Article}

Keywords: virus, bacteriophage, prophage, metagenomics, tool comparison

Posted Date: March 13th, 2021

DOI: https://doi.org/10.21203/rs.3.rs-287089/v1

License: (1) This work is licensed under a Creative Commons Attribution 4.0 International License.

Read Full License 


\title{
RESEARCH
}

\section{Simulation Study and Comparative Evaluation of Viral Contiguous Sequence Identification Tools}

\author{
Cody Glickman ${ }^{1,2^{*}}$, Jo Hendrix ${ }^{1,2}$ and Michael Strong ${ }^{1,2}$
}

\begin{abstract}
Background:

Viruses, including bacteriophage, are important components of environmental and human associated microbial communities. Viruses can act as extracellular reservoirs of bacterial genes, can mediate microbiome dynamics, and can influence the virulence of clinical pathogens. It is essential, therefore, to have robust sequence analysis methods in place to detect and annotate viral elements within microbial communities. Various targeted metagenomic analysis techniques detect viral sequences, but these methods often exclude large and genome integrated viruses. In this study, we evaluate and compare the ability of nine state-of-the-art bioinformatic tools, including Vibrant, VirSorter, VirSorter2, VirFinder, DeepVirFinder, MetaPhinder, JGI Earth Virome Pipeline, Kraken 2, and VirBrant, to identify viral contiguous sequences (contigs) across simulated metagenomes with different read distributions, taxonomic compositions, and complexities.

\section{Results:}

Of the tools tested in this study, VirSorter achieved the best F1 score while Vibrant had the highest average F1 score at predicting integrated prophages. Though less balanced in its precision and recall, Kraken2 had the highest average precision by a substantial margin.

We introduced the machine learning tool, VirBrant, which demonstrated an improvement in average F1 score over tools such as MetaPhinder. The tool utilizes machine learning with both protein compositional and nucleotide features. The addition of nucleotide features improves the precision and recall compared to the protein compositional features alone.

Viral identification by all tools was not impacted by underlying read distribution but did improve with contig length. Tool performance was inversely related to taxonomic complexity and varied by the phage host. Rhizobium and Enterococcus phage were identified consistently by the tools; whereas, Neisseria phage were commonly missed in this study.

\section{Conclusion:}

This study benchmarked the performance of nine state-of-the-art bioinformatic tools to identify viral contigs across different simulation conditions. This study explored the ability of the tools to identify integrated prophage elements traditionally excluded from targeted sequencing approaches. Our comprehensive analysis of viral identification tools to assess their performance in a variety of situations provides valuable insights to viral researchers looking to mine viral elements from publicly available metagenomic data.
\end{abstract}

Keywords: virus; bacteriophage; prophage; metagenomics; tool comparison

\footnotetext{
${ }^{*}$ Correspondence:

cody.glickman@cuanschutz.edu

${ }^{1}$ Center for Genes, Environment, and Health, National Jewish Health, 1400 Jackson Street, 80206 Denver, Colorado, USA Full list of author information is available at the end of the article
}

\section{Background}

Viruses are the most abundant biological entities on Earth [1]. However, the collective knowledge of environmental viral sequences, including bacteriophages, remains underrepresented relative to the amount of genetic information for eukaryotic viruses and bacteria. Bacteriophages are viruses that infect bacteria and are commonly referred to as phages. In addition to bacteria, phages are capable of infecting archaea. Phages are obligate parasites that play an important role in the genomic composition and evolution of their bacterial hosts. Phages directly contribute to 
bacterial infections in humans by acting as a genetic reservoir for virulent genes in bacteria such as Escherichia coli, Salmonella enterica, Pseudomonas aeruginosa, Vibrio cholerae, Corynebacterium diphtheriae, and Streptococcus pyogenes [2, 3].

In addition, some phages utilize Ig-like domains to attach to mucosal layers in humans to lie in wait for bacterial prey. This bacteriophage adherence to mucus (BAM) model suggests that phages may act as a non-host derived innate immunity system to modulate the bacterial microbiome [4]. A longitudinal study of the human virome revealed composition conservation that mimicked the stability of healthy bacterial microbiomes $[5,6]$. Dysbiosis in the virome has been observed in disease states such as inflammatory bowel disease (IBD), Crohn's disease, and asthma [7-9].

The study of viruses has traditionally relied on the ability to cultivate viral particles from a cultured host; however, many bacteria cannot be cultured in a laboratory setting [10]. The limited number of culturable hosts, in combination with the additional complexities of viral isolation limit the study of viruses. The advancements in next generation sequencing technologies created an opportunity to study viruses with culture independent methods. However, because viruses do not share a common universal marker gene, like the bacterial small subunit RNA, sequencing techniques such as metagenomics are a necessity [11]. Metagenomics is a non-targeted sequencing approach to elucidate the totality of genetic material within a sample, either DNA or RNA. However, in part due to small genomes, viruses are traditionally underrepresented in metagenomic studies from a read abundance perspective. It is common for viral reads to comprise less than $5 \%$ of metagenomic sequences [12]. A way to enrich viral reads in metagenomic studies is to filter or directly select viral like particles (VLPs). However, these techniques tend to remove large viruses and viruses integrated into bacterial genomes called prophages, before sequencing. Therefore, the ability to identify viral elements directly from metagenomic sequencing studies is also important for understanding the composition of the virome. The advent of computational tools dedicated to the identification of viral sequences in metagenomics has improved our ability to identify known, novel, and integrated viruses.

MetaPhinder is an approach that uses BLASTn and average nucleotide identity thresholds to classify viral contigs in metagenomics [13]. Methods that use sequence similarity suffers worsening performance with smaller contig lengths. Domain recognition is utilized by more tools to counter the limitations of contig length on traditional sequence homology approaches, but these tools are often reliant on specialized viral domains like those from pVOGs (prokaryotic virus orthologous groups) [14]. Unlike prophage identification methods that use viral domain enrichment or presence/absence to calculate a score, a new method, called Vibrant, uses domain abundances in a neural network framework to classify contigs having more than 4 proteins [15]. VirSorter2 follows a similar methodology, using domain percentages, protein compositionality features, and key homology genes in a tree-based machine learning framework to classify viral reads [16].

Homology of viral protein domains is limited to known viruses, which are thought to represent only a small slice of the vast viral dark matter [17]. Another homology approach sought to expand known viral hidden Markov models (HMMs) through a semi-supervised expansion of existing viral protein families. Paez-Espino 
et al. (JGI Earth Virome Pipeline) collected viral coding regions from NCBI servers and known viral metagenomic contigs; then clustered those peptides into protein families to create new viral HMMs [18]. This initial set was used as bait to identify potential viral contigs in thousands of metagenomic data sets. Predicted proteins from these captured viral contigs were added to the original set of peptides and reclustered to create thousands of new viral protein families and HMMs. Even with the expansion of viral families, both VirSorter and the JGI Earth Virome Pipeline are at least partially reliant on domain homology. A reference-free viral identification tool was developed using machine learning to address limitations of homology searching. VirFinder is a logistic regression classification using nucleotide sequence 8-mers as features [19]. The authors of VirFinder expanded the concept of using machine learning to identify viral contiguous sequences with DeepVirFinder, a convolutional neural network that takes raw sequences as inputs and learns features that are useful for viral contig prediction [20]. VirFinder relies solely on sequencebased features, which is analogous to another k-mer approach, Kraken2. Kraken2 uses discriminatory 35-mers to uniquely identify sequences to the species and even subspecies level [21]. In order to use Kraken2 in a viral identification context, we created the tool, VirKraken, that parses the Kraken2 classification output to identify viral contigs in metagenomic reads. VirKraken is available on PyPI and at https://github.com/Strong-Lab/VirKraken. VirKraken references the Kraken2 assigned taxonomy identification number against an edited NCBI Taxonomy database to assign kingdom and to filter sequences when requested [22].

Another approach to identify viral elements in metagenomics involves negation of known bacteria contigs. VirMine uses a homology search against a bacterial protein database; if hits of bacterial genes outnumber the number of unknown hits the contig is removed, thus leaving viral contigs [23]. All previously described tools identify viral elements from assembled sequences. MARVEL is a machine learning method that classifies binned reads as viral clusters using a random forest approach with three features (gene density, strand shifts, and fraction of homology hits to a viral protein database) [24]. We developed a machine learning model called VirBrant that uses both protein compositional features such as gene density and strand shift frequency, in addition to sequence-based features to classify viral contigs. The addition of protein compositional features is hypothesized to offset the dip in performance of sequence-based machine learning models compared to homology methods on longer contigs [19].

Many approaches exist to identify viral elements in metagenomics. However, a systemic evaluation among many of these tools has not been performed. This study is meant to provide information and guidance to researchers regarding when to use a specific viral identification tool to further study viral elements or to remove them for downstream analyses. The characterization of more viral elements in the public domain could lead to the discovery of novel viruses [25] and provide insight into the functional potential residing in an extracellular genetic reservoir [2].

\section{Methods}

VirBrant, a hybrid protein composition and nucleotide feature set for viral classification

To build VirBrant, 1,849 complete phage and 2,327 complete archaeal/bacterial genomes were compiled from RefSeq (Accessed on January 8th, 2020). Prophage 
elements in the archaeal and bacterial genomes were identified using VirSorter [22]. Category 4 prophages were selected, and the predicted nucleotide sequences were added to the complete viral genomes. Custom scripts were used to identify and remove the predicted prophage sequences from the host genome. The total number of prophages predicted was 730 in 339 bacterial genomes (14.57\% of genomes contained at least 1 prophage) culminating in an average prophage per genome ratio of 0.314 .

After removing integrated prophages, the complete genomes were fragmented into k-mers of 4 sizes using an n-step kmerization method. The n-step method removes contig end-overlap and ensures that the maximum number of k-mers is the length of the base sequence over the length $\mathrm{k}$. The complete genomes were fragmented into sizes of $1 \mathrm{~KB}, 3 \mathrm{~KB}, 5 \mathrm{~KB}$, and $10 \mathrm{~KB}$ sequences. Due to the size of bacterial and archaeal genomes relative to phage genomes, the fragments from non-phage sampling were down sampled to evenly distribute the classes. The four different fragment lengths were used to train four separate models.

The n-step fragments were subjected to a sliding window kmerization of size 8 using a k-mer counting program written in C [26]. A sliding window kmerization calculates k-mer abundance with significant overlap and the maximum number of $\mathrm{k}$-mers is the length of the base sequences minus 1 . The program stores all 8-mer values $(65,536$ possible 8 -mers) in a hash table. In real world metagenomic sampling, the directionality of a sequence fragment may be ambiguous. Therefore, similar to VirFinder [19], we developed custom scripts to sum complement, reverse, and reverse complement sequences thus reducing our feature space from 65,536 possible k-mers to 16,384 possible $\mathrm{k}$-mers. The nucleotide feature space is further reduced to 888 k-mers using Gini importance or total decrease in node impurity above 0.001, which is a weighted probability of reaching a feature averaged over all trees in a random forest [27].

\section{Protein composition feature set creation}

The use of protein compositional features is built into tools such as MARVEL and VirSorter [24, 28]. MARVEL and VirSorter both utilize gene density as a marker of viral elements. In this study, four protein features associated with viral genomes were included as part of the feature set in VirBrant; gene density, operon length, average peptide length, and percentage of overlapping peptides. Due to the physical restraints of some viral capsids, viral genomes are commonly tightly packed and translate shorter proteins than bacterial genomes [29]. In addition, viral genomes often have overlapped genes for different life cycles and have long stretches of genes located on the same strand [30]. Custom scripts were used to calculate the four protein characteristics from the output of the Prodigal gene prediction software [31]. Figure 1 shows the observed distribution of protein features in the training data of the $10 \mathrm{~KB}$ model.

\section{[Figure 1]}

\section{Model and hyperparameter selection}

After combining the complementary nucleotide features and the protein compositional features, the total feature space of VirBrant was 892 features. During 
training, the performance of a random forest, multi-layer perceptron, and an additive boosting model were compared using 5 -fold cross validation [32]. At every fragment size, the additive boosting model performed the best. We selected XGBoost (version 0.81) and performed a RandomSearchCV (version 0.20.1) analysis to determine hyperparameters $[32,33]$. The pre-trained models were added to the tool repository for use classifying metagenomic fasta sequences. VirBrant generates outputs as a header file containing the header sequences of viral elements and a fasta file containing the nucleotide information of the predicted viral elements.

\section{Building simulated Illumina metagenomes}

To build a simulated test set, all complete genomes were downloaded from NCBI RefSeq (accessed on 12/15/2020). The genomes deposited since May 1st, 2020 , were selected to test the viral contig identification tools because many of the tools were trained or relied on databases last updated prior to this date. Bacterial hosts of phages were collected using a dataset from Virus-Host DB [34] (Accessed on December 17, 2020). Phage were assigned bacteria genera values by their host organism. Using information from the Earth Microbiome Project (EMP) and from Qiita, the recently submitted genomes were further filtered by 53 genera commonly found in soils ( 37 genera) and in clinical samples (26 genera) with 8 genera in both niches [35, 36]. This resulted in 297 unique bacterial genomes being used for the simulations with 82 genomes found in both clinical sampling (160 genomes) and soil sampling (219 genomes). The reliance on recently submitted genomes to produce the testing set did not produce traditional bacterial distributions seen in clinical and soil microbiomes. For example, while the genera Bacteroides are commonly present in the clinical microbe samples, the amount in this study does not represent a substantial portion of the community as seen in other clinical microbiome studies [37]. The distribution of bacterial genera was used as a confounder for viral classification in this study. The goal of this study was to observe the performance of phage identification in the presence of genetically similar bacteria.

Phage genomes were also filtered by their host bacterial genera and randomly down sampled to match the number of bacterial genomes in the simulations. While phages are thought to outnumber bacteria ten to one in the environment [38], we matched the complexity of phage and bacteria in our simulations across taxonomic levels due to limitations in the number of available phage genomes for the full datasets. In order to test the impact of taxonomic complexity on viral identification tool performance, we subsampled phage and bacterial genomes into medium (50 bacterial genomes and 50 phage genomes) and low (10 bacterial genomes and 10 phage genomes) complexity subsets. Supplementary Table 1 (clinical) and Supplementary Table 2 (soil) detail the taxonomic abundance of the top 6 genera and phage host genera in the testing set across taxonomic complexity levels. While both lower complexities draw from the full distribution of genomes, there is no overlap in the selected genomes between the medium and low taxonomic levels. This was accomplished through setting a random seed in the subsampling procedure and using set operations to confirm no overlap of genomes.

Simulated metagenomes were created using InSilicoSeq (version 1.2.0). InSilicoSeq and another popular metagenomic simulator, CAMISIM use a lognormal read 
distribution by default, however, four additional read distributions are provided as a part of the InSilicoSeq software suite: uniform, exponential, zero inflated lognormal, and halfnormal $[39,40]$. Due to the enormous diversity of naturally occurring communities, read distribution profiles are likely to fluctuate. To understand the impact of read distribution and taxonomic complexities on the performance of viral identification, we created 30 MiSeq simulations with 12 million $2 \times 300$ reads. The 30 simulations were composed of two environmental conditions (clinical and soil microbes) with five read distributions across three taxonomic levels (full, medium, low). Bacterial reads represented $93.75 \%$ of the total composition in each simulation and phages represented $6.25 \%$. Prior studies suggest phages commonly represent less than $5 \%$ of metagenomic sequencing reads [12] due to genomes that are orders of magnitude smaller than prokaryotic genomes. Our decision to exceed the $5 \%$ of viral reads in metagenomics was driven by the need to identify an expanded set of phages from taxonomically diverse testing sets. After assembly and filtration of contigs less than $1 \mathrm{~KB}$ in length, phages comprise an average of $1.54 \%$ of total contig abundance.

After simulating, the reads were perfectly binned by sequence origin to limit the creation of chimeric contigs. Chimeric contigs are assembly errors when reads from different organisms are assembled together resulting in a shorter fragmented assembly or taxonomic misclassification downstream. The decision to bin prior to assembly was to allow for genera labeled contigs in order to explore false positive and recall rates of bacteria and phage, respectively. The perfect bins were assembled using metaSpades (version 3.11.1) and only contigs of length $1 \mathrm{~KB}$ or greater were retained [41]. The relative abundance of bacteria genera in the simulations are shown in Figure 2.

\section{[Figure 2]}

\section{Integrated prophage identification}

Integrated prophage elements were identified in complete bacterial genomes using VirSorter prior to read simulations [28]. Integrated prophages were selected for downstream processing if assigned as category 4 , the highest confidence category for prophages within VirSorter [28]. A nucleotide BLAST database was created with the identified prophage elements. After read simulation and assembly, bacterial contigs were identified as prophages using a BLASTn search against the prophage database with a bitscore greater than 1000 and a percent identity greater than $95 \%$. Supplementary Figure 1 shows the genera distribution of the identified prophage elements separated by read distribution and sampling site.

Tools used in simulation study

\section{[Table 1]}

The tools used in the study shown in Table 1 were tested on their performance to identify viral elements from assembled contigs in the simulations. The tools used in this study to identify viral contigs were Vibrant (Version 1.2.0), VirSorter, 
VirSorter2, VirFinder, DeepVirFinder, MetaPhinder, JGI Earth Virome Pipeline, Kraken 2, and VirBrant [15, 16, 19, 28].

Any VirSorter predictions that were classified to the lowest confidence category were removed via evidence by the tool developers [28]. VirFinder and DeepVirFinder assign a probability value and any contigs that had a value less than 0.01 were classified as viral. A diamond blast database was created with the viral proteins from the JGI Earth Virome Pipeline [18, 42]. Proteins from the simulation contigs were predicted using Prodigal and searched for viral homology using diamond BLAST against proteins from the JGI Earth Virome Pipeline with matches retained that had a bit score greater than 100 and an e-value less than 1e-05 [31]. Contigs with more than one hit were classified as viral. MetaPhinder, VirBrant, VirBrant Proteins, and Vibrant were run with default parameters [13, 15]. VirSorter2 uses the include groups flag to capture both double-stranded DNA phage and single stranded DNA viruses specific to phage as described by the authors [16]. Kraken 2 was run with default parameters using the minikraken database from March 2020 [21]. The resulting Kraken 2 report was parsed for viral reads using VirKraken (Version 0.0.5).

\section{Tool performance scoring}

The structure of the simulation allowed for each contig to possess a true origin label. These labels were used to identify the performance of the tools to identify viral elements in the simulations. The performance was measured by precision, recall, and F1 score. Prophages were considered viral in this study and an additional analysis of tool performance on prophage identification was performed. The performance measures were used in a simulation performance ranking system to determine the best performing tool across different scenarios. The performance of each tool was ranked within each condition with 1 representing the best performing tool. The highest-ranking value (worst performing tool) changes as some tools were unable to properly calculate a score. This occurs when a tool did not predict any viral element in a simulation.

In addition to overall performance, tool performance is evaluated at four discretized contig lengths: $1 \mathrm{~KB}-2.5 \mathrm{~KB}, 2.5 \mathrm{~KB}-5 \mathrm{~KB}, 5 \mathrm{~KB}-10 \mathrm{~KB}, 10 \mathrm{~KB}+$. The recall of the tools to identify viral elements by genera was used to determine any systematic biases for or against specific viral groups. Visualizations of scoring metrics were performed in Python using a combination of Matplotlib (version 2.2.3) and Seaborn (version 0.9.0) plotting software [43, 44]. Kruskal-Wallace nonparametric testing was performed to determine if the scoring values arose from the same distributions.

\section{Results}

\section{Overall tool performance}

The F1 performance across different read simulation conditions was not significantly different $(\mathrm{H}=4.02, \mathrm{p}=0.404$, Kruskal-Wallis). The $\mathrm{F} 1$ performance was significantly different by taxonomic complexity with tool performance in lower complexity simulations enriched relative to both medium and full complexity simulations ( $\mathrm{H}=47.65, \mathrm{p}=4.50 \mathrm{e}-11$, Kruskal-Wallis). The F1 performance, as well as precision and recall, of longer contigs specially the $10 \mathrm{~KB}+$ bin was enriched relative to other contig length bins $(\mathrm{H}=275.7, \mathrm{p}=1.82 \mathrm{e}-59$, Kruskal-Wallis). Table 
2 contains the mean performance of the tools and the average ranking across the 30 simulations. The F1 performance of the tools in the simulation discretized by taxonomic complexity is shown in Figure 3.

\section{[Table 2]}

\section{[Figure 3]}

Kraken2 led both average precision and precision rank. In this study, Earth Virome led in recall and recall rank. The tool with the highest average F1 score and best F1 rank was VirSorter. VirSorter was also the tool used to perform prophage identification. This may provide VirSorter with an advantage over other tools in prophage identification.

\section{Prophage identification performance}

The prophage performance of the low complexity simulations are removed due to the presence of only a single prophage contig in all 10 simulations. The F1 performance of the tools to identify prophage in 20 medium and high complexity simulations is shown in Table 3.

\section{[Table 3]}

\section{Tool performance by contig length}

As the length of the contigs increase, the performance of the tools improved. Figure 4 demonstrates the F1 performance of each tool within defined contig length bins. If the F1 score of a tool was 0 , the record was removed as some simulations lack shorter contiguous sequences.

\section{Viral recall by host genera}

Recall scores of viral elements from the medium and full distributions were calculated across 30 host genera. Recall was only retained if greater than 0 to prevent the absence of a phage host genera by niche. Figure 5 shows the recall of viral contigs by host genera across all tools. The viral host genera with the best recall was Xanthomonas, however, phage with Xanthomonas as a host were not well represented in the data set. Phage known to infect Enterococcus achieved an average recall over 0.83 across all tools. DeepVirFinder performed the best at identifying phage known to infect Enterococcus with an average recall rate of 0.97. Neisseria phage had the lowest average recall performance across all tools (0.23), with only 7 tools correctly predicting at least one Neisseria phage contig. The Earth Virome performed the best at identifying this elusive phage (0.68) and the next best tool was MetaPhinder with a recall rate of 0.24 . 


\section{False positive genera}

In addition to the recall rate of viral elements by host genera, the percent of genera associated with bacterial false positives was calculated for each tool in medium and full complexity simulations. Bacterial genera that represent more than one third of false positives of a tool in a simulation were retained. Eleven genera were represented with Streptomyces present in 9 of 10 tools. Additionally, Citrobacter and Pseudomonas were major false positive genera in more than 5 tools. Supplemental Figure 2 shows the genera of false positives that represent more than $33 \%$ by tool.

\section{Discussion}

This study benchmarked and evaluated the ability of nine viral classification tools to identify viral and prophage elements within shotgun metagenomics. The study consisted of 30 Illumina MiSeq simulations across two communities, five read abundance distributions, and three taxonomic levels. The performance of the tools was consistent across read distributions ( $\mathrm{H}=4.02, \mathrm{p}=0.404$, Kruskal-Wallis), whereas, the average performance increased with a reduction in taxonomic complexity $(\mathrm{H}=47.65, \mathrm{p}=4.50 \mathrm{e}-11$, Kruskal-Wallis). Lower taxonomic complexity was associated with longer contig lengths in the assemblies and longer contigs were associated with improved overall performance.

The differences between performance scores suggests the selection of a tool may depend upon the desired application. VirSorter scored the highest average F1 score and had the best F1 ranking across all the simulations. Kraken2 may be the ideal tool when minimizing the number of false positives. The Earth Virome pipeline had the best recall; however, the application of this tool is not meant for traditional viral identification due to the large false positive rate. The Earth Virome protein set was derived from an iterative viral protein domain search and may include many unknown proteins that may not truly be derived from viral sources [18]. Even so, the broad homology search space still failed to capture all viral derived contigs demonstrating the difficulty of viral identification within metagenomic samples.

Prophage identification in metagenomics is a difficult problem as many integrated viral elements are degraded in bacterial hosts to drive evolution [45]. As such, remnants of prophage particles are scattered across bacterial genomes and viral genes can be mistakenly attributed as bacterial in origin. Many tools to identify prophages in whole genome experiments fail to generalize to metagenomics due to fragmentation that breaks down traditional viral enrichment measurements. The decision to select the highest confidence prophage predictions using VirSorter from the complete genomes prior to simulation may have provided VirSorter with an added performance boost. Vibrant had the highest average F1 score and best F1 ranking at identifying prophages across all 20 simulations. Kraken 2 had the highest average precision and VirSorter had the best precision ranking. The Earth Virome proteins exceled at recall; however, the next best tools were VirFinder and DeepVirFinder. VirFinder and DeepVirFinder like many other tools that perform well with prophage recall have a high false positive rate.

The performance of all tools would increase with an additional step of removing known bacterial contigs. One approach is to search for genes unique to bacteria and archaea, the 16S rRNA. 16S rRNA profiles from RFAM can be applied to the 
RNA domain search tool, Infernal, to remove contigs with known bacterial genes $[46,47]$. This approach may impact the recovery of prophage contigs if the integration site of the virus was near a $16 \mathrm{~S}$ rRNA.

Viral identification tools performed well at identifying phages known to infect genera such as Enterococcus (0.83), Mycobacterium (0.77), and Salmonella (0.81). The performance of the tools to identify phages that infect genera such as Neisseria (0.23), Brevibacterium (0.30), and Mesorhizobium (0.33) dropped substantially. Detecting the presence of Neisseria phage may be important for a diagnostic of invasive meningococcal disease as prophage-like elements are commonly found throughout the Neisseria genera [48].

The performance of VirBrant including the nucleotide features showed improvement over the protein compositional features alone. The precision of VirBrant dramatically improved with contigs over $10 \mathrm{~KB}$, however, smaller bins were plagued with many false positives. Integrated prophages added to the viral class in the training data represented $28.3 \%$ of the total viral genomes. Prophages are commonly degraded in bacterial hosts to drive evolution [45], therefore degraded viral elements in bacterial contigs with similar nucelotide structures as the complete prophages may be misclassified. In addition, the use of k-mer profiles for smaller contig classification created sparse data sets, which may have led to overfitting.

The performance of the tools presented needs to be weighted with the computational cost to run each tool. This study was performed on a shared high performance computing cluster and individual tool performance and memory requirements were not captured on an isolated node. However, the mechanism of viral identification can be used infer the relative time and memory consumption of the tools. The fastest tool in this study was Kraken2, which uses discriminatory k-mers to compare against a pre-computed hash table. The amount of memory needed to build the full hash table may be a drawback against using Kraken2 on a personal machine. The machine learning tool, Vibrant, uses protein features derived from multiple HMM searches. As a result of a large domain space, this tool ran for a significantly longer amount of time (1 week for full complexity simulations) relative to the other tools on the shared compute cluster.

This study benchmarked and compared the performance of viral identification tools in metagenomics. The viral identification performance measures, in conjunction with the genera and prophage recall, highlights the advantages and challenges of using specific viral identification tools, and can be used as a guide to assist the selection of tools for subsequent research.

\section{Conclusion}

In summary, we tested the performance of nine viral identification tools on 30 simulated metagenomes. The underlying read distribution has little impact on average tool performance. Increasing contig length and decreasing taxonomic complexity improved the average performance of the tools. Vibrant performed the best at the identification of prophages in metagenomics. Overall, the tool that averaged the best F1 score was VirSorter, while Kraken2 lead all other tools in precision. The results of these simulations should provide researchers with a guide to selecting the appropriate tool for their own viral identification research. 


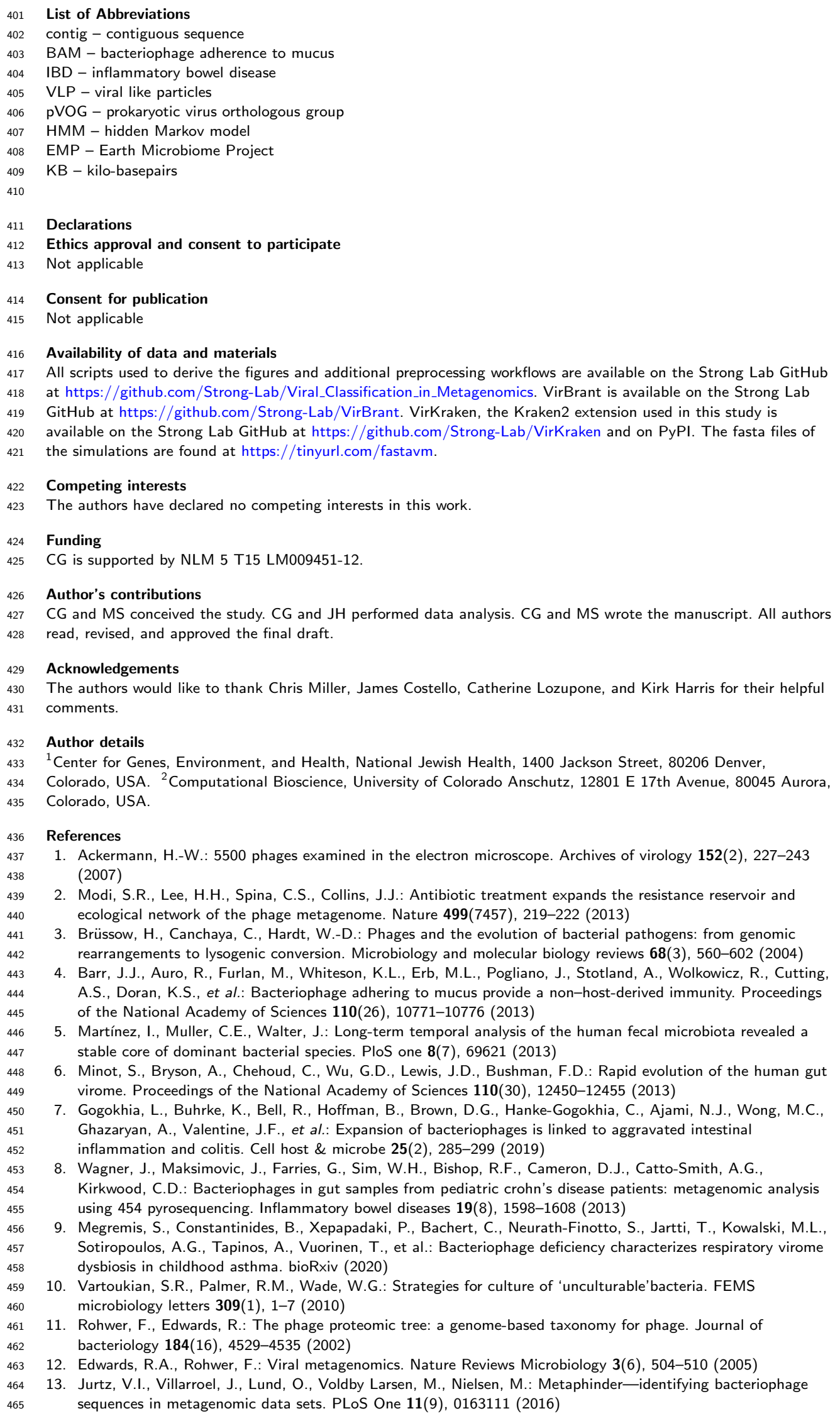


14. Grazziotin, A.L., Koonin, E.V., Kristensen, D.M.: Prokaryotic virus orthologous groups (pvogs): a resource for comparative genomics and protein family annotation. Nucleic acids research, 975 (2016)

15. Kieft, K., Zhou, Z., Anantharaman, K.: Vibrant: automated recovery, annotation and curation of microbial viruses, and evaluation of viral community function from genomic sequences. Microbiome 8(1), 1-23 (2020)

16. Guo, J., Bolduc, B., Zayed, A.A., Varsani, A., Dominguez-Huerta, G., Delmont, T.O., Pratama, A.A., Gazitúa, M.C., Vik, D., Sullivan, M.B., et al.: Virsorter2: a multi-classifier, expert-guided approach to detect diverse dna and rna viruses. Microbiome 9(1), 1-13 (2021)

17. Martínez-García, M., Santos, F., Moreno-Paz, M., Parro, V., Antón, J.: Unveiling viral-host interactions within the 'microbial dark matter'. Nature communications 5(1), 1-8 (2014)

18. Paez-Espino, D., Eloe-Fadrosh, E.A., Pavlopoulos, G.A., Thomas, A.D., Huntemann, M., Mikhailova, N., Rubin, E., Ivanova, N.N., Kyrpides, N.C.: Uncovering earth's virome. Nature 536(7617), 425-430 (2016)

19. Ren, J., Ahlgren, N.A., Lu, Y.Y., Fuhrman, J.A., Sun, F.: Virfinder: a novel k-mer based tool for identifying viral sequences from assembled metagenomic data. Microbiome 5(1), 69 (2017)

20. Ren, J., Song, K., Deng, C., Ahlgren, N.A., Fuhrman, J.A., Li, Y., Xie, X., Poplin, R., Sun, F.: Identifying viruses from metagenomic data using deep learning. Quantitative Biology, 1-14 (2020)

21. Wood, D.E., Lu, J., Langmead, B.: Improved metagenomic analysis with kraken 2. Genome biology 20(1), 1-13 (2019)

22. Federhen, S.: The ncbi taxonomy database. Nucleic acids research 40(D1), 136-143 (2012)

23. Garretto, A., Hatzopoulos, T., Putonti, C.: virmine: automated detection of viral sequences from complex metagenomic samples. PeerJ 7, 6695 (2019)

24. Amgarten, D., Braga, L.P., da Silva, A.M., Setubal, J.C.: Marvel, a tool for prediction of bacteriophage sequences in metagenomic bins. Frontiers in genetics 9, 304 (2018)

25. Dutilh, B.E., Cassman, N., McNair, K., Sanchez, S.E., Silva, G.G., Boling, L., Barr, J.J., Speth, D.R., Seguritan, V., Aziz, R.K., et al.: A highly abundant bacteriophage discovered in the unknown sequences of human faecal metagenomes. Nature communications 5(1), 1-11 (2014)

26. Alex Reynolds: Kmer-counter. https://github.com/alexpreynolds/kmer-counter

27. Breiman, L., Friedman, J., Stone, C.J., Olshen, R.A.: Classification and Regression Trees. CRC press, ??? (1984)

28. Roux, S., Enault, F., Hurwitz, B.L., Sullivan, M.B.: Virsorter: mining viral signal from microbial genomic data PeerJ 3, 985 (2015)

29. Hatfull, G.F., Jacobs-Sera, D., Lawrence, J.G., Pope, W.H., Russell, D.A., Ko, C.-C., Weber, R.J., Patel, M.C. Germane, K.L., Edgar, R.H., et al.: Comparative genomic analysis of 60 mycobacteriophage genomes: genome clustering, gene acquisition, and gene size. Journal of molecular biology 397(1), 119-143 (2010)

30. Hatfull, G.F., Cresawn, S.G., Hendrix, R.W.: Comparative genomics of the mycobacteriophages: insights into bacteriophage evolution. Research in microbiology 159(5), 332-339 (2008)

31. Hyatt, D., Chen, G.-L., LoCascio, P.F., Land, M.L., Larimer, F.W., Hauser, L.J.: Prodigal: prokaryotic gene recognition and translation initiation site identification. BMC bioinformatics 11(1), 1-11 (2010)

32. Pedregosa, F., Varoquaux, G., Gramfort, A., Michel, V., Thirion, B., Grisel, O., Blondel, M., Prettenhofer, P., Weiss, R., Dubourg, V., et al:: Scikit-learn: Machine learning in python. the Journal of machine Learning research 12, 2825-2830 (2011)

33. Chen, T., Guestrin, C.: Xgboost: A scalable tree boosting system. In: Proceedings of the 22nd Acm Sigkdd International Conference on Knowledge Discovery and Data Mining, pp. 785-794 (2016)

34. Mihara, T., Nishimura, Y., Shimizu, Y., Nishiyama, H., Yoshikawa, G., Uehara, H., Hingamp, P., Goto, S., Ogata, H.: Linking virus genomes with host taxonomy. Viruses 8(3), 66 (2016)

35. Thompson, L.R., Sanders, J.G., McDonald, D., Amir, A., Ladau, J., Locey, K.J., Prill, R.J., Tripathi, A., Gibbons, S.M., Ackermann, G., et al.: A communal catalogue reveals earth's multiscale microbial diversity. Nature 551(7681), 457-463 (2017)

36. Gonzalez, A., Navas-Molina, J.A., Kosciolek, T., McDonald, D., Vázquez-Baeza, Y., Ackermann, G., DeReus, J., Janssen, S., Swafford, A.D., Orchanian, S.B., et al.: Qiita: rapid, web-enabled microbiome meta-analysis. Nature methods 15(10), 796-798 (2018)

37. Wexler, H.M.: Bacteroides: the good, the bad, and the nitty-gritty. Clinical microbiology reviews 20(4), 593-621 (2007)

38. Labrie, S.J., Samson, J.E., Moineau, S.: Bacteriophage resistance mechanisms. Nature Reviews Microbiology 8(5), 317-327 (2010)

39. Gourlé, H., Karlsson-Lindsjö, O., Hayer, J., Bongcam-Rudloff, E.: Simulating illumina metagenomic data with insilicoseq. Bioinformatics 35(3), 521-522 (2019)

40. Fritz, A., Hofmann, P., Majda, S., Dahms, E., Dröge, J., Fiedler, J., Lesker, T.R., Belmann, P., DeMaere, M.Z., Darling, A.E., et al.: Camisim: simulating metagenomes and microbial communities. Microbiome 7(1), $1-12(2019)$

41. Nurk, S., Meleshko, D., Korobeynikov, A., Pevzner, P.A.: metaspades: a new versatile metagenomic assembler. Genome research 27(5), 824-834 (2017)

42. Buchfink, B., Xie, C., Huson, D.H.: Fast and sensitive protein alignment using diamond. Nature methods 12(1), 59-60 (2015)

43. Hunter, J.D.: Matplotlib: A 2d graphics environment. IEEE Annals of the History of Computing 9(03), 90-95 (2007)

44. Waskom, M., the seaborn development team: Mwaskom/seaborn. doi:10.5281/zenodo.592845. https://doi.org/10.5281/zenodo.592845

45. Bobay, L.-M., Touchon, M., Rocha, E.P.: Pervasive domestication of defective prophages by bacteria Proceedings of the National Academy of Sciences 111(33), 12127-12132 (2014)

46. Griffiths-Jones, S., Bateman, A., Marshall, M., Khanna, A., Eddy, S.R.: Rfam: an rna family database. Nucleic acids research 31(1), 439-441 (2003)

47. Nawrocki, E.P., Eddy, S.R.: Infernal 1.1: 100-fold faster rna homology searches. Bioinformatics 29(22), 
2933-2935 (2013)

48. Al Suwayyid, B.A., Rankine-Wilson, L., Speers, D.J., Wise, M.J., Coombs, G.W., Kahler, C.M.: Meningococcal disease-associated prophage-like elements are present in neisseria gonorrhoeae and some commensal neisseria species. Genome biology and evolution 12(2), 3938-3950 (2020) 
Figures

Figure 1: Protein compositional feature perfomance The performance of the 4 protein compositional features in the $10 \mathrm{~KB}$ training dataset. A) Gene density represented by number of genes per $1 \mathrm{~KB}$. B) Median operon length is a representative measure of strand switching frequency. An operon is defined as as a set of closely linked genes on the same strand. C) Percentage of overlapping peptides measured as a percentage of all predicted genes. Viruses that peptides are commonly shorter than bacterial peptides.

Figure 2: Relative abundance of genera in simulations These figures highlight the relative abundance of the contigs greater than $1 \mathrm{~KB}$. Bacterial contigs represent $98.46 \%$ of contigs, while phages and prophages combine for the

Figure 3: F1 scores of tools by taxonomic conditions

554 Dodge boxplots by taxonomic complexity arranged by average F1 performance with the best performing tools on the 555 right side of the $x$ axis.

556 Figure 4: F1 scores of tools across contig length bins in all simulations

557 The average F1 performance of all tools increases as the bin representing contig lengths increases. All thirty

558 simulations are included as part of this figure, however in some simulations, predicted viral contigs of a specific

559 length are absent. This may cause some tools to have more data points than others.

560 Figure 5: Viral recall by host genera in medium and full complexity simulations

561 The 30 host genera of phage are listed in order of mean recall along the $x$-axis. The dotted line in the figure

562 demarcates 0.5 recall. 
Table 1: Tools used in viral identification benchmarking study

\begin{tabular}{|c|c|c|c|c|c|c|}
\hline Tool & Last updated & Target & $\begin{array}{l}\text { Viral } \\
\text { homology matching }\end{array}$ & $\begin{array}{l}\text { Compositional } \\
\text { protein features }\end{array}$ & $\begin{array}{l}\text { Machine } \\
\text { learning classification }\end{array}$ & $\begin{array}{l}\text { Programming } \\
\text { skills required }\end{array}$ \\
\hline VirSorter & 2015 & Virus & Yes & Yes & No & No \\
\hline VirSorter2 & 2020 & Virus & Yes & Yes & Yes & No \\
\hline VirFinder & 2017 & Virus & No & No & Yes & Yes \\
\hline DeepVirFinder & 2020 & Virus & No & No & Yes & Yes \\
\hline Vibrant & 2020 & Virus & Yes & No & Yes & Yes \\
\hline MetaPhinder & 2016 & Phage & Yes & No & No & Yes \\
\hline Earth Virome & 2020 & Virus & Yes & No & No & Yes \\
\hline VirBrant & 2020 & Phage & No & Yes & Yes & Yes \\
\hline Kraken2+VirKraken & 2020 & Virus & Yes & No & No & Yes \\
\hline
\end{tabular}

Table 2: Average performance and simulation rankings of tools at identifying phage

\begin{tabular}{|l|l|l|l|l|l|l|}
\hline Tool & F1 Rank & Precision Rank & Recall Rank & Average F1 & Average Precision & Average Recall \\
\hline VirSorter & $\mathbf{2 . 1 0}$ & 3.10 & 6.40 & $\mathbf{0 . 6 3 6}$ & 0.640 & 0.658 \\
\hline Kraken2 & 2.93 & $\mathbf{1 . 0 7}$ & 7.80 & 0.609 & $\mathbf{0 . 9 6 2}$ & 0.467 \\
\hline Vibrant & 3.52 & 4.10 & 7.26 & 0.560 & 0.573 & 0.598 \\
\hline VirFinder & 3.93 & 2.30 & 9.32 & 0.548 & 0.717 & 0.450 \\
\hline DeepVirFinder & 5.04 & 5.38 & 7.90 & 0.432 & 0.392 & 0.496 \\
\hline VirSorter2 & 5.27 & 5.93 & 3.10 & 0.463 & 0.341 & 0.797 \\
\hline VirBrant & 5.40 & 6.00 & 3.60 & 0.413 & 0.317 & 0.755 \\
\hline VirBrant Proteins & 7.47 & 7.70 & 4.63 & 0.142 & 0.213 & 0.717 \\
\hline MetaPhinder & 8.73 & 8.67 & 2.83 & 0.082 & 0.138 & 0.044 \\
\hline Earth Virome & 9.73 & 9.73 & $\mathbf{1 . 7 8}$ & 0.023 & 0.872 & $\mathbf{0 . 8 7 2}$ \\
\hline
\end{tabular}

Table 3: Average performance and simulation rankings of tools at identifying prophage

\begin{tabular}{|l|l|l|l|l|l|l|}
\hline Tool & F1 Rank & Precision Rank & Recall Rank & Prophage F1 & Prophage Precision & Prophage Recall \\
\hline Vibrant & $\mathbf{1 . 1 5}$ & 1.95 & 7.45 & $\mathbf{0 . 1 6 9}$ & 0.146 & 0.231 \\
\hline VirSorter & 2.11 & $\mathbf{1 . 6 6}$ & 8.76 & 0.147 & 0.144 & 0.164 \\
\hline VirSorter2 & 2.70 & 3.40 & 4.70 & 0.117 & 0.0685 & 0.453 \\
\hline VirBrant & 3.95 & 4.45 & 7.13 & 0.0446 & 0.0269 & 0.252 \\
\hline VirBrant Proteins & 5.20 & 5.50 & 6.05 & 0.0188 & 0.00978 & 0.342 \\
\hline Kraken2 & 6.70 & 1.85 & 9.90 & 0.0169 & $\mathbf{0 . 1 7 2}$ & 0.00896 \\
\hline MetaPhinder & 6.65 & 6.85 & 2.90 & 0.0152 & 0.00776 & 0.588 \\
\hline Earth Virome & 6.85 & 7.00 & $\mathbf{1 . 6 0}$ & 0.0117 & 0.00588 & $\mathbf{0 . 7 2 8}$ \\
\hline VirFinder & 8.88 & 8.88 & 2.73 & 0.00725 & 0.00365 & 0.705 \\
\hline DeepVirFinder & 8.79 & 9.03 & 2.79 & 0.00647 & 0.00325 & 0.637 \\
\hline
\end{tabular}




\section{Figures}

\section{Figure 1}

A

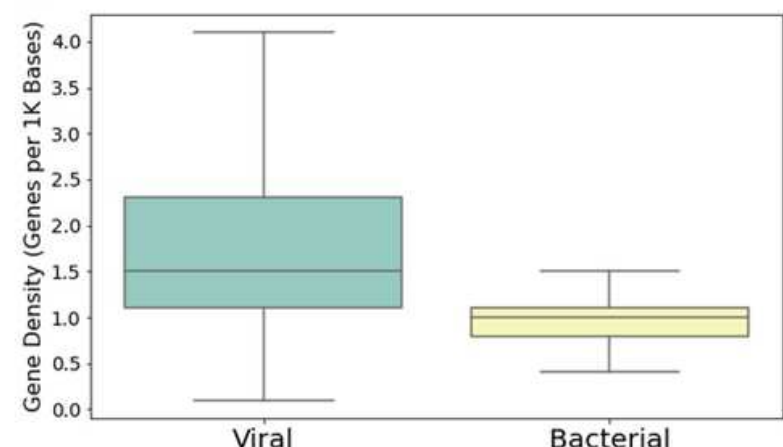

C

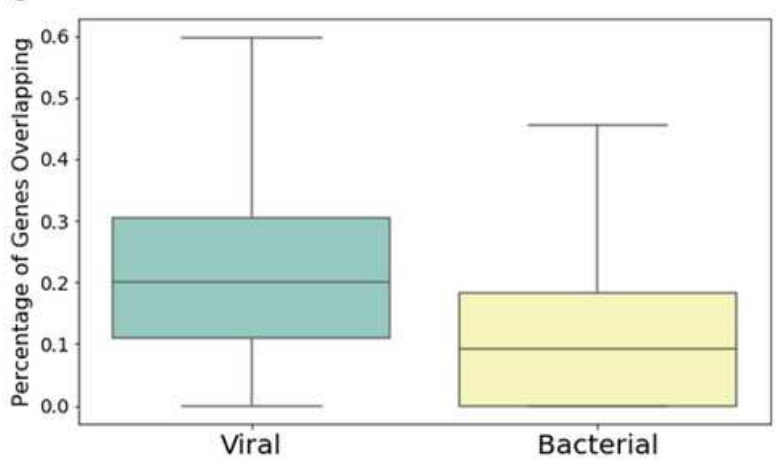

B

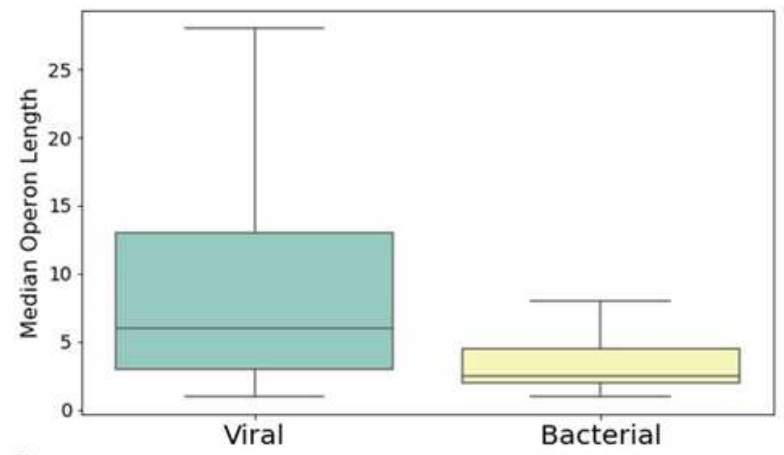

D

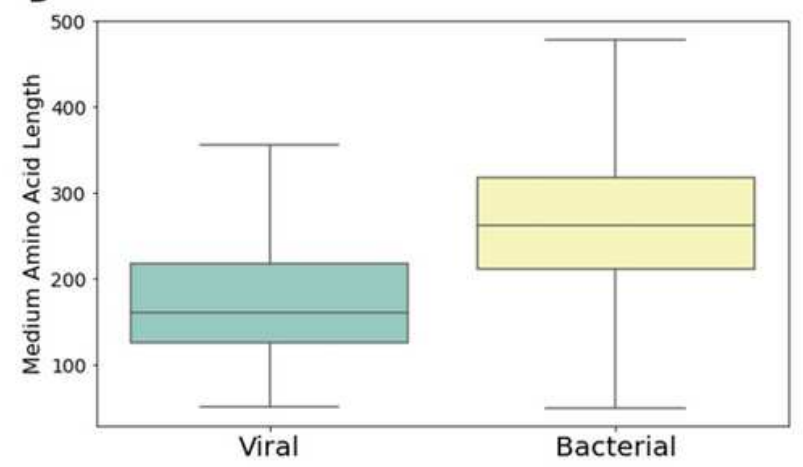

\section{Figure 1}

Protein compositional feature performance The performance of the 4 protein compositional features in the $10 \mathrm{~KB}$ training dataset. A) Gene density represented by number of genes per $1 \mathrm{~KB}$. B) Median operon length is a representative measure of strand switching frequency. An operon is dened as as a set of closely linked genes on the same strand. C) Percentage of overlapping peptides measured as a percentage of all predicted genes. Viruses that have a lysogeny phase are known overlap genes for different life cycles. D) Median amino acid length as viral peptides are commonly shorter than bacterial peptides. 


\section{Figure 2}

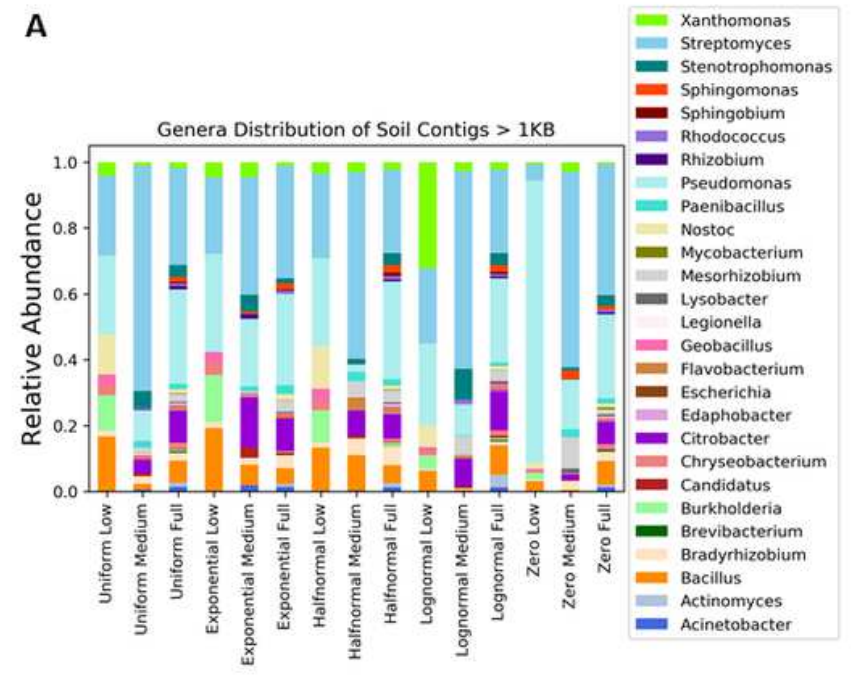

Simulation Condition

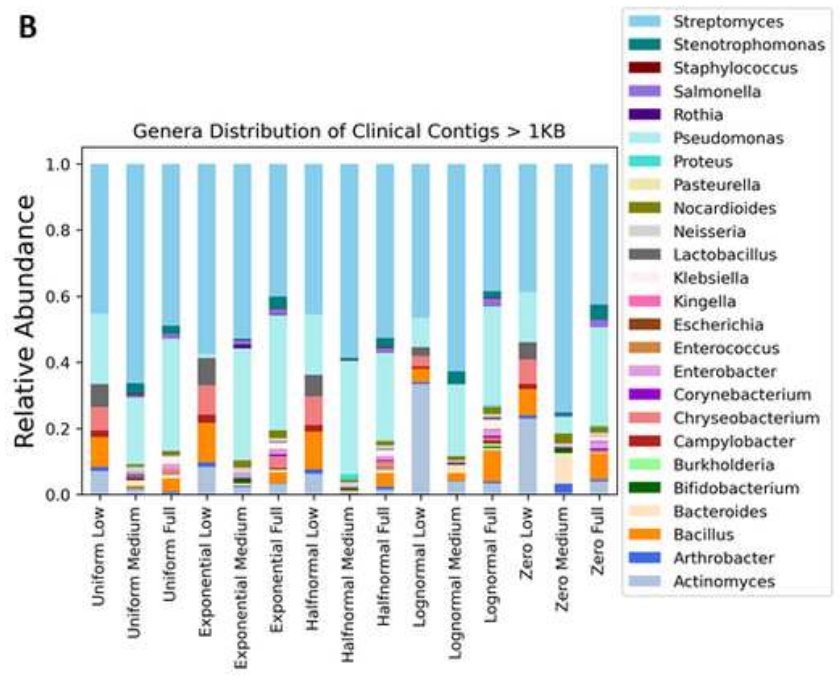

Simulation Condition

\section{Figure 2}

Relative abundance of genera in simulations These figures highlight the relative abundance of the contigs greater than $1 \mathrm{~KB}$. Bacterial contigs represent $98.46 \%$ of contigs, while phages and prophages combine for the remaining $1.54 \%$. A) The contig distribution within 15 soil simulations. B) The contig distribution within 15 clinical simulations. 


\section{Figure 3}

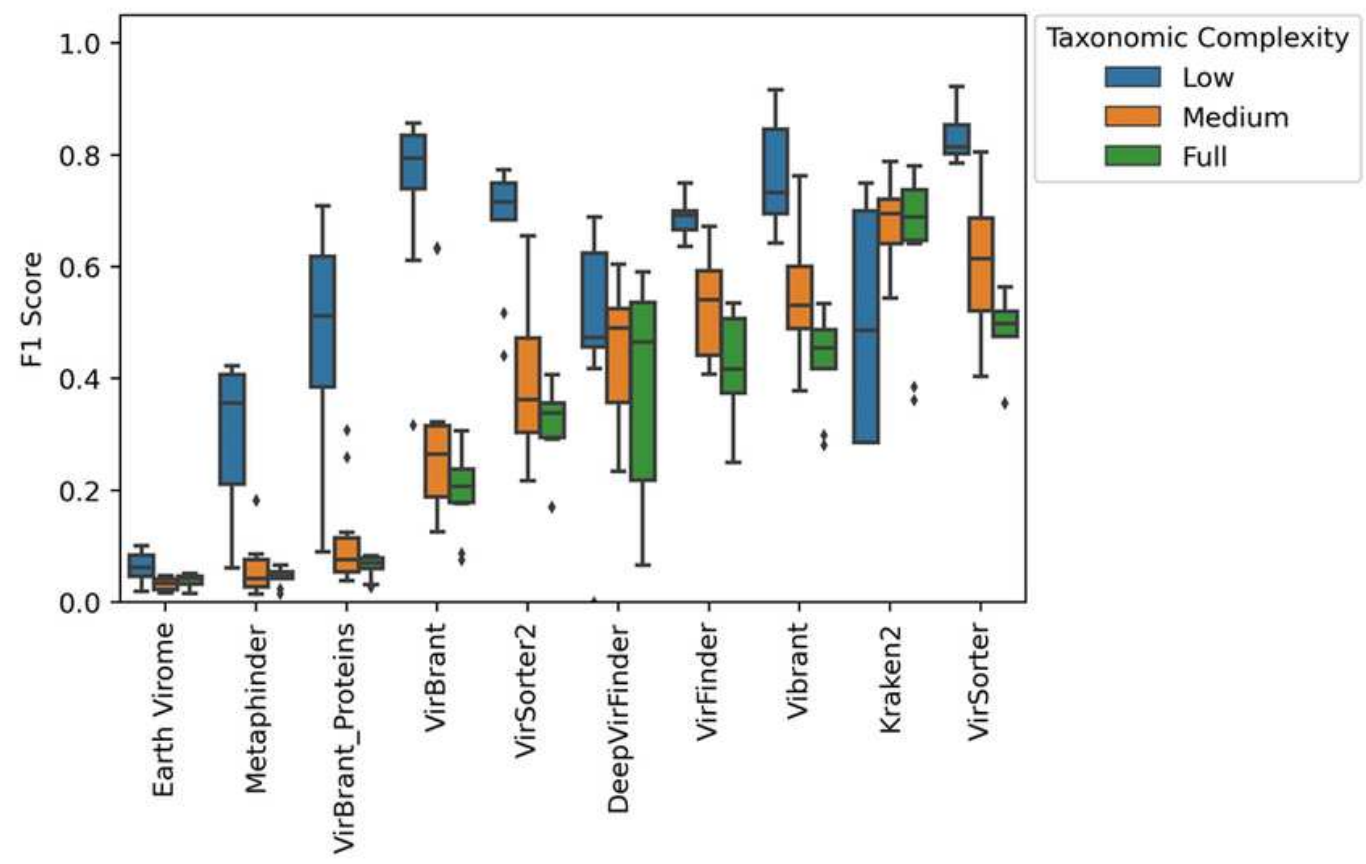

Figure 3

F1 scores of tools by taxonomic conditions Dodge boxplots by taxonomic complexity arranged by average $\mathrm{F} 1$ performance with the best performing tools on the right side of the $\mathrm{x}$ axis.

\section{Figure 4}

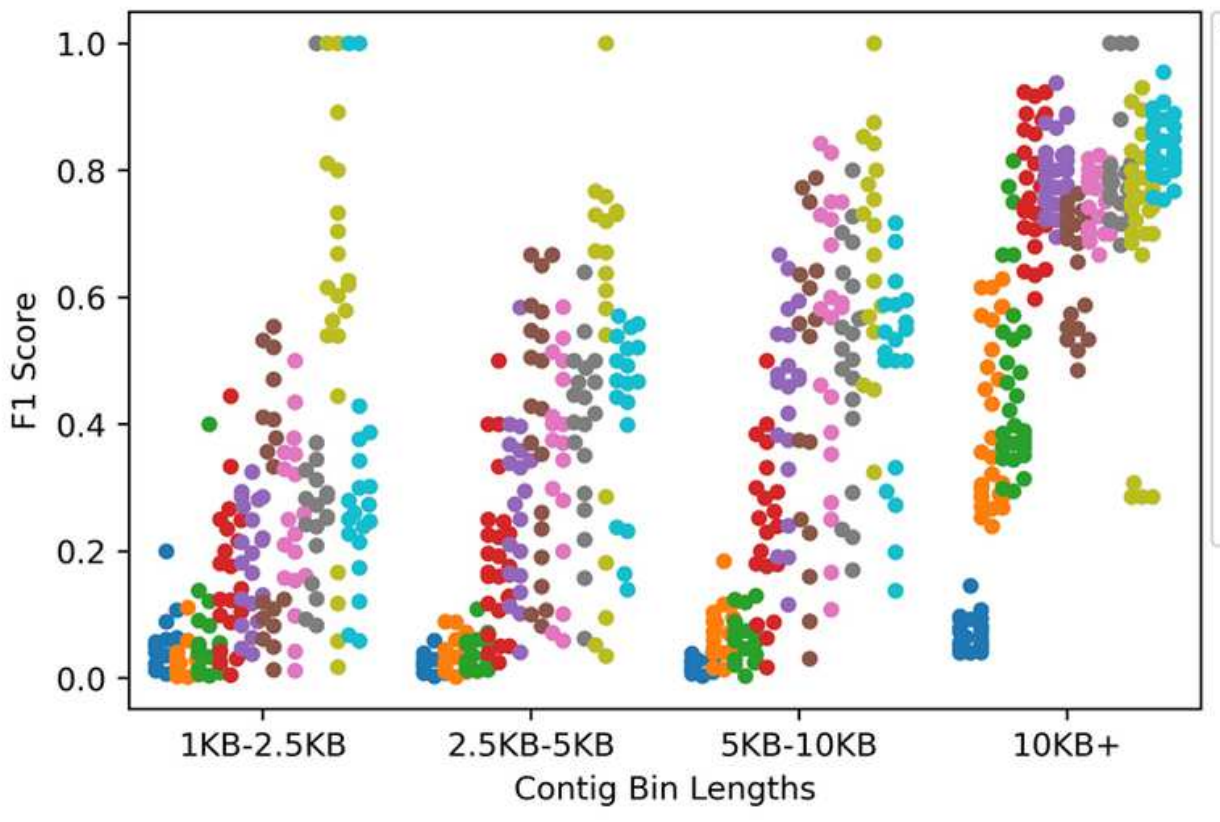
Viral Identification Tools
- Earth Virome
Metaphinder
VirBrant_Proteins
- VirBrant
VirSorter2
- DeepVirFinder
- VirFinder
Vibrant
Kraken2
VirSorter


Figure 4

F1 scores of tools across contig length bins in all simulations The average F1 performance of all tools increases as the bin representing contig lengths increases. All thirty simulations are included as part of this figure, however in some simulations, predicted viral contigs of a specific length are absent. This may cause some tools to have more data points than others.

Figure 5

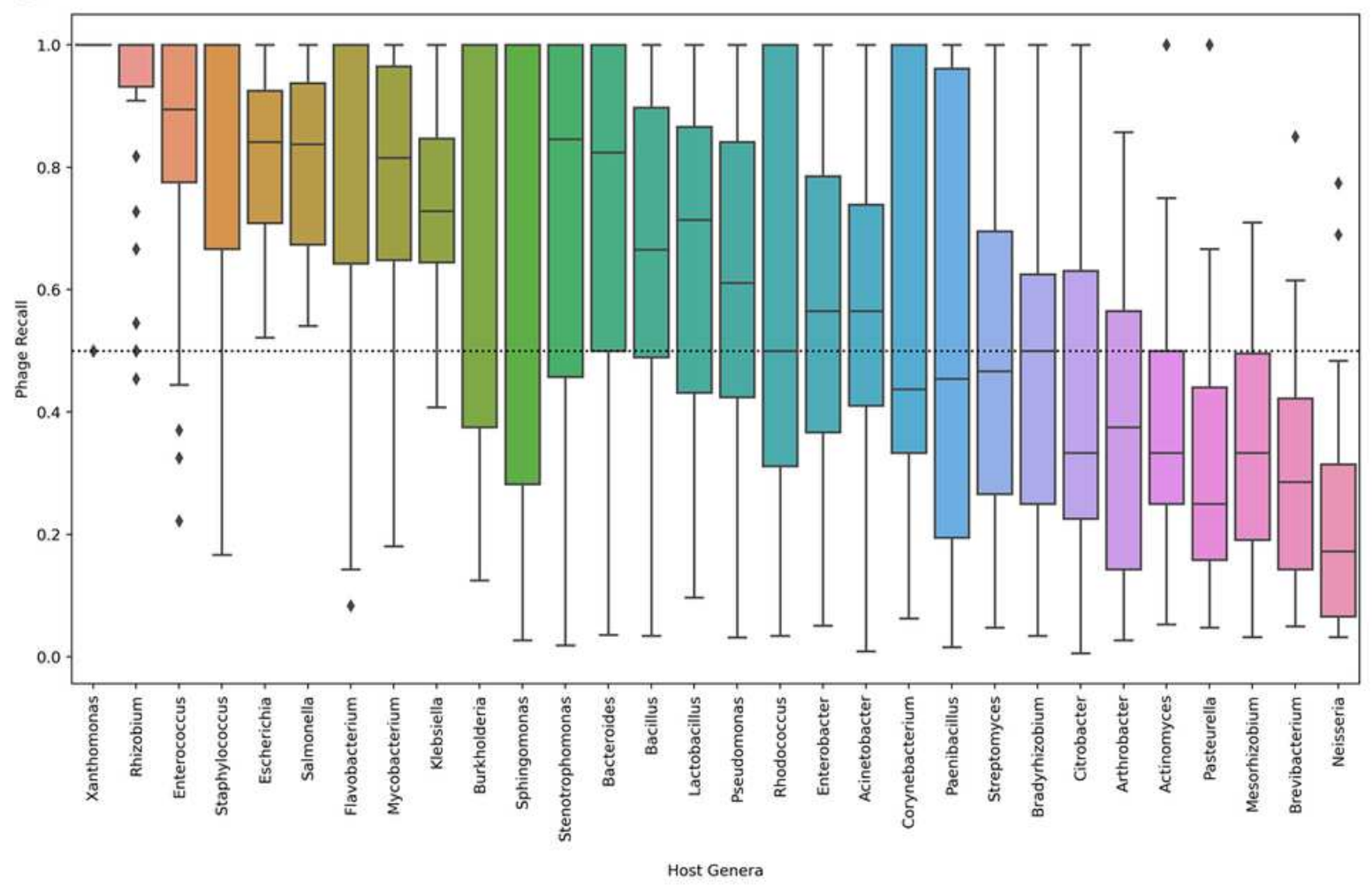

Figure 5

Viral recall by host genera in medium and full complexity simulations The 30 host genera of phage are listed in order of mean recall along the x-axis. The dotted line in the figure demarcates 0.5 recall.

\section{Supplementary Files}

This is a list of supplementary files associated with this preprint. Click to download.

- GlickmanBMCSupplemental.pptx

- SupplementalGlickmanBMC.pdf 